\section{Vitreous surgery with direct central retinal artery massage for central retinal artery occlusion}

N Lu, N-L Wang, GL Wang, XW Li and Y Wang
Although multiple therapeutic regimens have been proposed for it, the effect seems to be limited. The visual prognosis for most patients was poor. Visual acuity increased by three or more lines in only $15 \%$ of the patients with or without treatment. ${ }^{1}$ Selective intra-arterial lysis has been proposed for the treatment of CRAO in recent years. A meta-analysis of the published data showed that there may be marginal visual benefit, and it has potential serious adverse effects, such as embolization of other parts of the vascular system, which might lead to stroke or even death. ${ }^{2}$ In this study, vitreous surgery with direct central retinal artery massage as a new technique for the treatment of CRAO was investigated. To our knowledge, there have been no similar reports in the literature previously.

Patients and methods

\section{Patients}

This study was approved by the Committee Review Board of Beijing Tongren Hospital. It consisted of 10 consecutive patients with acute CRAO who were treated with vitreous surgery with direct central retinal artery massage between October 2005 and April 2007 in Beijing Tongren Hospital. Informed consent was obtained from all the patients before treatment.

All the eyes included in our study showed fundus features typical of acute CRAO, with abrupt painless visual loss. Criteria of exclusion: CRAO of more than 6 days' duration at initial examination; patients with a foveal sparing cilioretinal artery; the caliber of the retinal vessels had returned to normal and angiographic images revealed a normal retinal circulation time; initial visual acuity better than counting fingers.
Ophthalmology Center of Beijing Tong-Ren Hospital, Capital Medical University, Beijing, China

Correspondence: N-L Wang, Ophthalmology Center of Beijing Tong Ren Hospital, Capital Medical University, Dong Jiao Min Xiang No.1, Beijing 100730, China Fax: +65131244.

E-mail: luningnl@ yahoo.com.cn

Received: 15 June 2007 Accepted in revised form: 31 March 2008 Published online: 16 May 2008

\section{Competing interest} statement: There are no proprietary or commercial interests related to the article

Previous presentation: The article is partly presented at the 7th EURETINA Congress in Monte Carlo 17-20 May, 2007 
Before the surgery, best-corrected visual acuity, anterior and posterior segments of the eye were examined. Photography of the posterior pole of all the patients and fundus fluorescein angiography (FFA) in some patients were performed. Laboratory tests were conducted for blood clotting, electrolytes, and blood sugar.

Ten patients with an average CRAO period of $73 \mathrm{~h}$ ( $24 \mathrm{~h}$ to 6 days) were enrolled. The age of the patients ranged from 34 to 70 years, with an average of 48 years (Table 1). Nine patients had a history of arterial hypertension, in which, one had a history of stroke 2 years ago, and three had a history of myocardial infarction. These patients were under drug therapy for high blood pressure. Other history of special medical treatment was denied. Only one patient had no history of systemic diseases (Table 1). Temporal arteritis was excluded by examining the blood sedimentation rate.

Retinal edema with cherry red spot and narrowed retinal arteries were found in all the cases (Figure 1a). Initial visual acuity in these cases ranged from no light perception to counting fingers (Table 1). Emboli of the central retinal artery were not detected in these cases. Small yellow-white emboli were found in the retinal arterioles but not on the optic disc in two cases. FFA was performed in five patients. The arteriovenous transit times ranged from 18 to $311 \mathrm{~s}$, with an average of $138 \mathrm{~s}$.

\section{Technique of vitreous surgery with direct central retinal artery massage}

After a standard 3 port pars plana vitrectomy, a special probe made of nickel titanium and extended from a 19 gauge support shaft (Figure 2) was put on the central bifurcation of the retinal artery, and slight pressure was applied until entire flow cessation of the artery occurred, followed by $2 \mathrm{~s}$ of release. This was repeated for $5-6$ times. If small pieces of embolus were seen moving downstream from the central retinal artery and the caliber of the retinal vessels increased, then the operation could be finished. If no change of the retinal circulation occurred after this procedure, a microvitreoretinal blade was used to make a small incision along the central retinal artery in the optic nerve head, and then the special probe was introduced carefully into the incision and advanced into the optic nerve head toward the lamina cribrosa along the central retinal artery. The site of the incision was carefully selected to avoid major branches of the retinal arterioles and venules. As the probe moving toward the lamina cribrosa, slight pressure was applied against the central retinal artery until entire blood flow cessation occurred, followed by $2 \mathrm{~s}$ of release. This procedure was repeated for 5-6 times. Approximately 3-4 $\mathrm{mm}$ of the probe entered the optic nerve head. If the caliber of the retinal arteries increased, then the operation could be finished. If no change of the retinal circulation occurred following this procedure, then a sclera depressor was used to press the optic nerve head until slight deformation of the optic nerve head was observed under the surgical microscope, followed by $2 \mathrm{~s}$ of release. Again, this procedure was repeated for 5-6 times before the surgery was completed. This procedure was achieved by inserting a sclera depressor retrobulbarly through the superonasal conjunctival incision. The whole movement of the sclera depressor along the superonasal surface of the sclera toward the optic nerve head was observed under the surgical microscope.

\section{Patient follow-up}

Follow-up examinations were performed at $24 \mathrm{~h}, 48 \mathrm{~h}$, 1 week, 2 weeks, 3 weeks, 4 weeks and 2 months after

Table 1 Clinical characteristics of the patients with CRAO and visual changes before and after treatment

\begin{tabular}{rcclcllll}
\hline Patient no. & Sex & $\begin{array}{c}\text { Age } \\
\text { (years) }\end{array}$ & $\begin{array}{l}\text { Systemic } \\
\text { associations }\end{array}$ & $\begin{array}{c}\text { Occlusion } \\
\text { time }(h)\end{array}$ & Initial VA & Final VA & $\begin{array}{c}\text { Post-treatment } \\
\text { circulation }\end{array}$ & $\begin{array}{c}\text { Follow-up } \\
\text { time (days) }\end{array}$ \\
\hline 1 & M & 42 & HBP & 144 & HM & CF & Recovered & 30 \\
2 & M & 49 & HBP & 96 & HM & CF & Not recovered & 30 \\
3 & M & 38 & HBP & 72 & CF & $30 / 400$ & Recovered & 60 \\
4 & M & 38 & HBP & 24 & CF & $20 / 32$ & Recovered & 90 \\
5 & F & 52 & & 96 & HM & HM & Not recovered & 90 \\
6 & F & 53 & HBP and MI & 96 & CF & $30 / 400$ & Recovered & 60 \\
7 & M & 44 & HBP and MI & 24 & HM & $20 / 200$ & Recovered & 75 \\
8 & M & 44 & HBP & 36 & CF & $20 / 32$ & Recovered & 60 \\
9 & M & 70 & HBP and MI & 72 & HM & $20 / 200$ & Recovered & 30 \\
10 & M & 51 & HBP and stroke & 72 & NLP & HM & Recovered & 30 \\
\hline
\end{tabular}

CRAO indicates central retinal artery occlusion; VA, visual acuity; HM, hand movement; CF, counting fingers; LP, light perception; NLP, no light perception; HBP, high blood pressure; MI, myocardial infarction. 


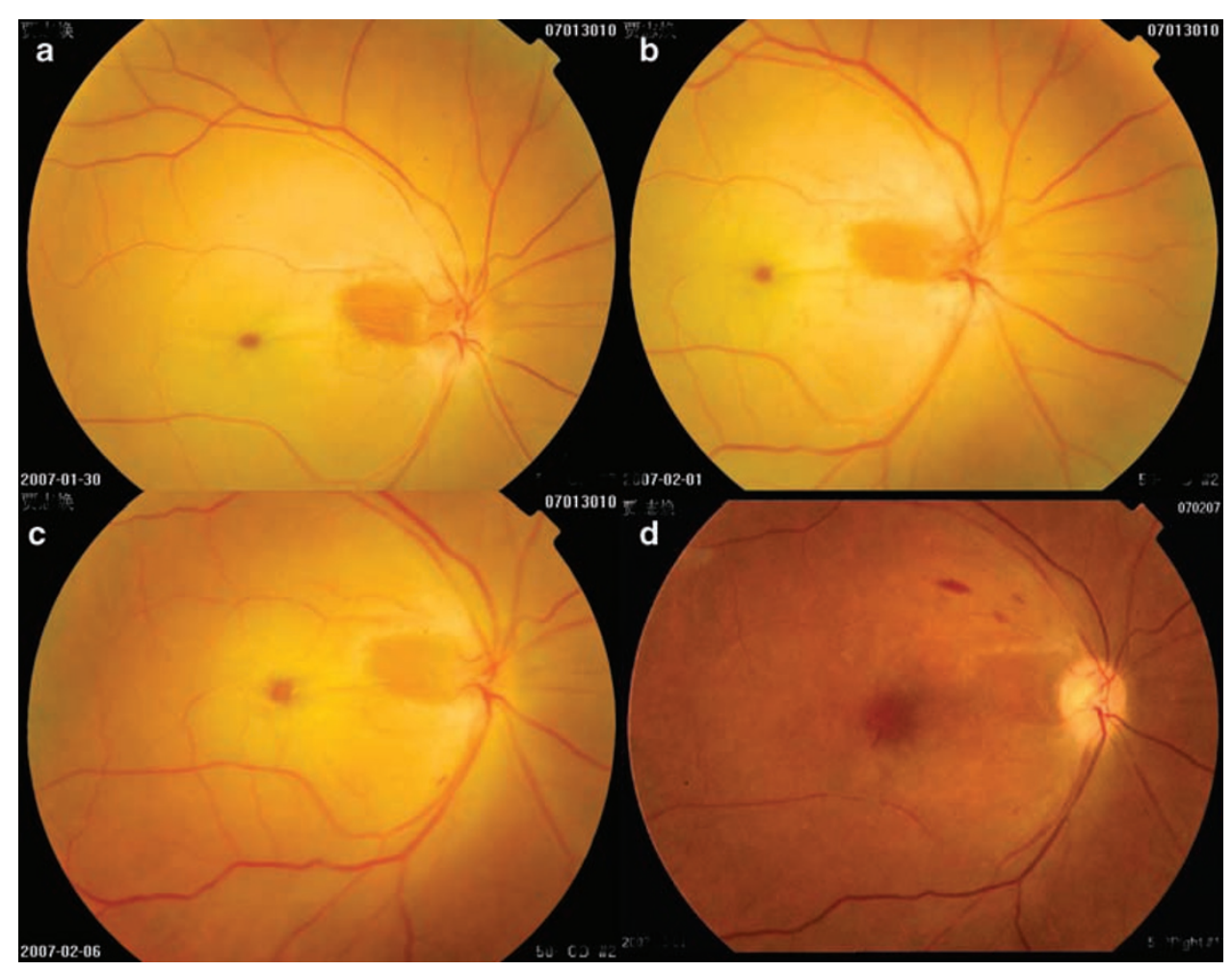

Figure 1 (a) Colored fundus photograph of a 44-year-old woman with central retinal artery occlusion for $24 \mathrm{~h}$ and visual acuity of hand movements. The retinal arterioles are severely narrowed, and there is heavy retinal edema. Small patch of normal looking retina was seen near the optic disc due to an efficient cilioretinal arteriole, but the macular was not spared. (b) First day after operation, the caliber of the retinal arterioles looks greater, especially at the macular region. (c) Fifth day after operation, the retinal vessels look normal and the retinal edema decreased prominently. (d) Thirty days later, although the retinal edema subsided and visual acuity improved to $20 / 200$, thinned retinal arterioles are also left with optic disc pallor.
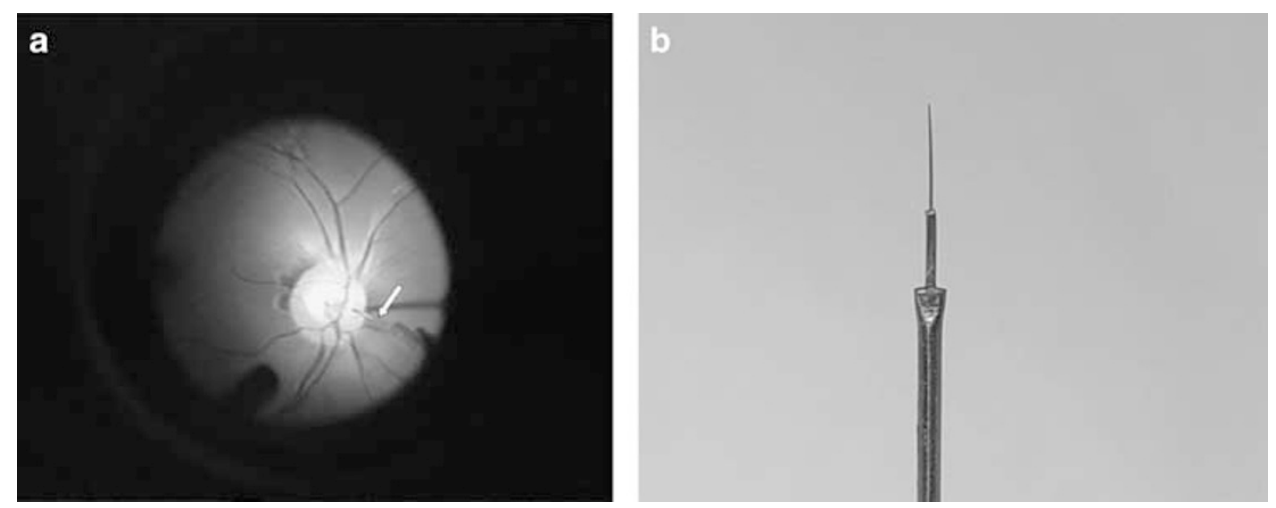

Figure 2 (a) Fundus photograph of a patient during operation. The arrow indicates the probe being used for direct central retinal artery massage. (b) Fundus photograph of the probe (patent has been obtained).

intervention. This included test of the best-corrected visual acuity, fundus photography, and FFA.

\section{Results}

Ten patients with acute CRAO were treated by vitreous surgery with direct central retinal artery massage. In four cases, small pieces of yellow-white embolus were seen dislodged from the central retinal artery and moved downstream along one branch of the retinal arterioles toward the periphery of the fundus following the direct central retinal artery massage. At the same time, the vessel caliber of both the retinal artery and vein increased, indicating that the retinal blood flow 
Table 2 Characteristics of the patients with CRAO during and after operation

\begin{tabular}{lccc}
\hline Characteristic & $\begin{array}{c}\text { All eyes } \\
(\mathrm{n}=10)\end{array}$ & $\begin{array}{c}\text { Circulation improved } \\
(\mathrm{n}=8)\end{array}$ & $\begin{array}{c}\text { Circulation not improved } \\
(\mathrm{n}=2)\end{array}$ \\
\hline $\begin{array}{l}\text { VA improved for } \geqslant 3 \text { lines } \\
\text { localized hemorrhage on the optic nerve head during }\end{array}$ & 6 & 6 & 0 \\
$\begin{array}{l}\text { operation } \\
\text { Embolus dislodged during operation }\end{array}$ & 9 & 7 & 2 \\
$\begin{array}{l}\text { Circulation improved during operation } \\
\text { Circulation improved 24-48 h after operation }\end{array}$ & 4 & 4 & 0 \\
$\begin{array}{l}\text { Postop arteriovenous transit times on FFA } \\
\text { Postop CRVO and NVI }\end{array}$ & 4 & 4 & 0 \\
\end{tabular}

CRAO indicates central retinal artery occlusion; VA, visual acuity; Postop, postoperative; FFA, fundus fluorescein angiography; CRVO, central retinal vein occlusion; NVI, neovascularization of the iris.

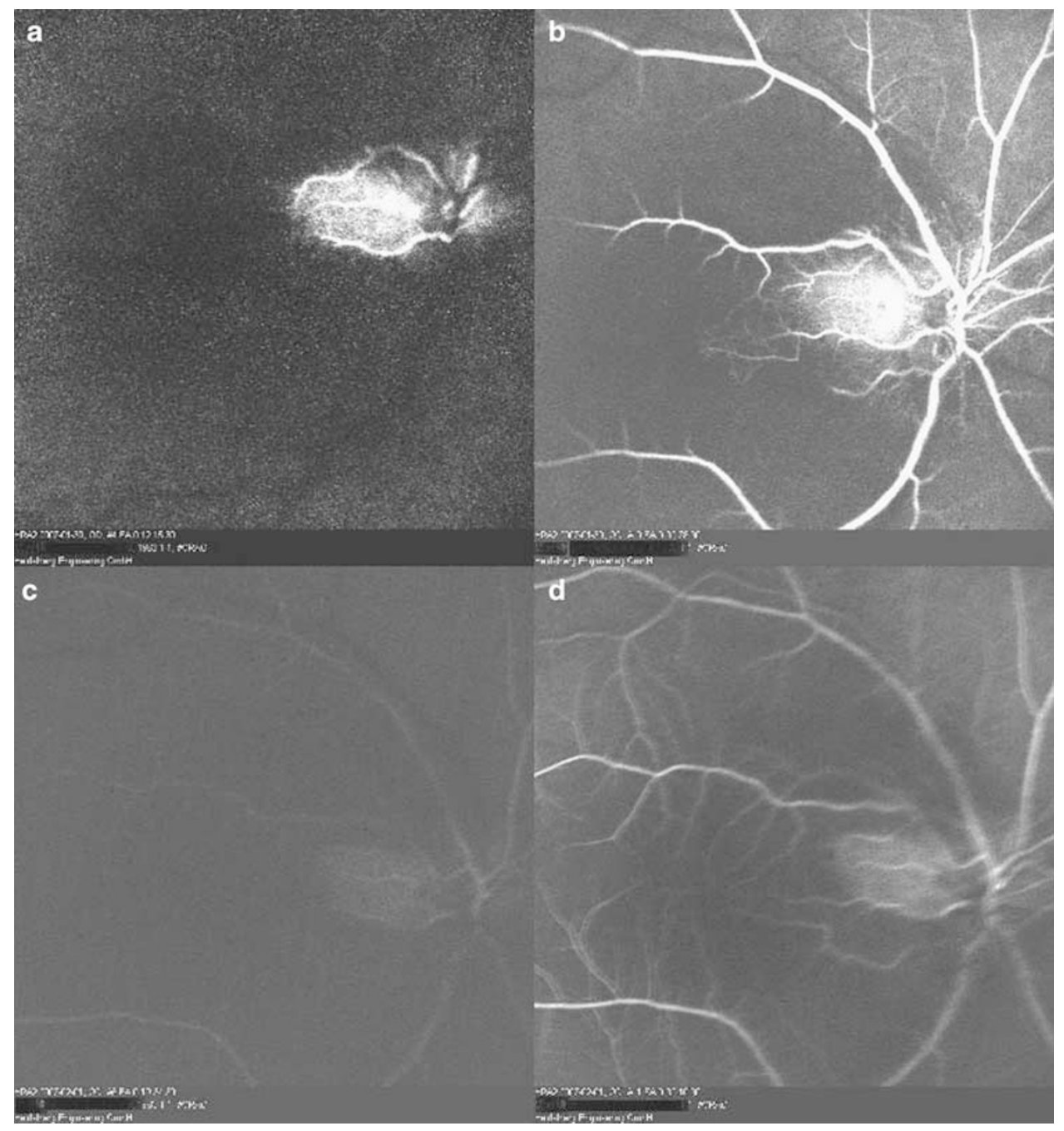

Figure 3 Fundus fluorescein angiograph of the same patient as in Figure 1. (a) Before operation, at $12 \mathrm{~s}$ after injection, the cilioretinal arterioles are filled with dye, but the central retinal artery is not filled yet. (b) Before operation, at $33 \mathrm{~s}$ after injection, there is retinal vascular hypoperfusion. (c) On the first day after operation, at $19 \mathrm{~s}$ after dye injection, the central retinal artery, retinal arterioles, and the cilioretinal arterioles are filled with dye. (d) On the first day after operation, at $32 \mathrm{~s}$ after injection, the retinal vessels are well filled with dye. 
improved. The central retinal artery became narrowed again 2 days after operation in one patient, but reopened on the third day postoperatively. In other six cases, no change of retinal circulation was found during the operation, among which, retinal circulation improved in four cases $24-48 \mathrm{~h}$ after operation, remained the same in two cases. The arteriovenous transit times on FFA performed within the first 2 days postoperatively were normal or nearly normal as shown in Table 2 (normal arteriovenous transit times were $\leqslant 11 \mathrm{~s}$ ). ${ }^{3}$ FFA was not performed for the two patients with no change in the ophthalmoscopic appearance. During follow-up visits, retinal artery occlusion did not recur in the eight cases with improved retinal circulation (Figures 1, 3 and 4). Visual acuity improvement occurred within the first week after operation in most patients. In some patients, visual acuity gradually improved during the first 2 months postoperatively (Table 1 ).

During the operation, localized retinal hemorrhage on the optic nerve head occurred in most cases due to injury of the capillaries at the operation site, which was controlled by increasing the infusion pressure.

Postoperative FFA showed slight staining of fluorescence at the operation site on the optic nerve head (Figure 4). In one case, central retinal vein occlusion occurred 5 days after operation. Iris neovascularization occurred 20 days later (Table 2). Cryotherapy of the peripheral retina for 360 degrees was performed and the neovascularization of the iris regressed gradually after operation. No other serious complication occurred.

\section{Discussion}

Clinical and experimental studies have indicated that there was a time limit for CRAO to cause irreversible damage to the retina. If retinal circulation restored within this time limit, retinal function could recover. In primates, the time limit was $105 \mathrm{~min} .{ }^{4}$ In the clinic, this time limit could be much longer, because the occlusion in CRAO is seldom complete. ${ }^{5}$ It was reported that vision recovered to different degrees within $12-33 \mathrm{~h}$ after CRAO. 5,6 There was even a report that showed that vision could recover after 4 days of CRAO. ${ }^{7}$ In our study, for all the patients with an occlusion time of 4 days or less, visual acuity improved in the cases that retinal blood circulation restored after surgery. For the patient with 6 days of occlusion, visual acuity remained the same although retinal circulation restored postoperatively

(Table 1). We cannot comment on the correlation between the occlusion time and the degree of visual recovery, as the number of cases is too small.

The common cause of CRAO is an embolus lodging in the central retinal artery. The actual position of the embolus is difficult to determine. It may be immediately

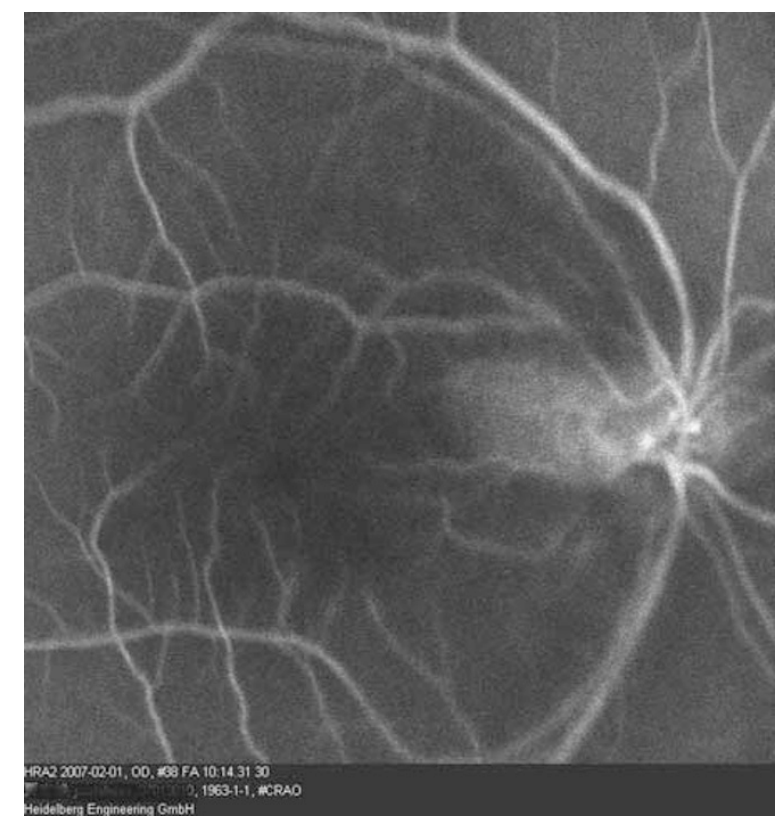

Figure 4 Late stage postoperative fluorescein angiograph of the same patient as in Figure 1. At $10 \mathrm{~min}$ after injection, there is slight staining of fluorescence at the operation site on the optic nerve head.

posterior to the lamina cribrosa ${ }^{5}$ or more posterior. ${ }^{8}$ In our study, pressing the central retinal artery in the optic nerve head with a probe or retrobulbarly with a sclera depressor led to deformation and bending of the blood vessel wall. The vessel wall of the retinal artery is elastic, therefore, slight deformation and bending may not cause any damage to the vessel wall. The emboli were made of cholesterol in $74 \%$, calcified material in $10.5 \%$, and platelet-fibrin in $15.5 \% .^{9}$ The emboli may be less flexible and more fragile in comparison with the wall of the retinal arteries, so during the mechanical movement of the vessels induced by the probe, the embolus may be broken into smaller pieces and then be dislodged. During the operation, emboli were seen dislodged and moving downstream with immediate restoration of retinal circulation after the direct central retinal artery massage in four of our cases, indicating that mechanical movement may be effective to dislodge the embolus. In the other four cases, no embolus was seen during the operation, but the retinal circulation improved $24-48 \mathrm{~h}$ postoperatively. This may be because the emboli were more adherent to the vessel wall or less fragile, which made it more difficult to be dislodged immediately. However, the direct retinal artery massage may had loosened the embolus and made it readily dislodged or absorbed gradually, so $24-48 \mathrm{~h}$ after operation, retinal circulation restored. For two cases, circulation did not recover, this may be due to that the position of the emboli 
was further posterior to the optic nerve head or there was no embolus at all.

The diameter of the probe was $0.1 \mathrm{~mm}$, similar to the diameter of the central retinal artery (Figure 2). It was advanced into the optic nerve head in a parallel direction to the optic nerve fiber, and was pressed directly upon the blood vessel wall, so it may cause little damage to the optic nerve. One day after operation, there was only slight staining at the operation sites on FFA, and most patients had an increased visual acuity during follow-up study (Figure 4). This indicated that the operation caused no or negligible damage to both the central retinal artery and the optic nerve. Only one patient developed central retinal vein occlusion 5 days after operation. This may be due to damage of the central retinal vein during the operation. As retinal circulation did not recover in this case during follow-up study, slow blood flow in the vein might gradually caused thrombus formation at the site of injury, and finally resulted in central retinal vein occlusion. This indicated that the operation had a potential risk of central retinal vein injury.

Visual acuity improved by three or more lines in 6 out of 10 cases $(60 \%)$. Forty percent of our patients had a final visual acuity of $20 / 200$ or better (Table 1 ). The visual outcome was better than that of conservative forms of treatment or natural course of the disease. ${ }^{1}$ Our study showed that vitreous surgery with direct central retinal artery massage was relatively safe and may be effective in restoring blood circulation in patients with CRAO and improving the visual prognosis. Limitations of our study included: noncomparative nature, limited number of patients, and delayed treatment due to patient referral. A randomized controlled trial is necessary to prove the effectiveness of the treatment.

\section{References}

1 Mueller AJ, Neubauer AS, Schaller U, Kampik A. Evaluation of minimally invasive therapies and rationale for a prospective randomized trial to evaluate selective intraarterial lysis for clinically complete central retinal artery occlusion. Arch Ophthalmol 2003; 121: 1377-1381.

2 Beatty S, Eong KGA. Local intra-arterial fibrinalysis for acute occlusion of the central retinal artery: a meta-analysis of the published data. Br J Ophthalmol 2000; 84: 914-916.

3 Brown GC, Magargal LE. Central retinal artery obstruction and visual acuity. Ophthalmology 1982; 89: 14-19.

4 Hayreh SS, Weingeist TA. Experimental occlusion of the central artery of the retina. IV: Retinal tolerance time to acute ischemia. Ophthalmology 1980; 64: 818-825.

5 Rumelt S, Dorenboim Y, Rehany U. Aggressive systemic treatment for central retinal artery occlusion. Am J Ophthalmol 1999; 128: 733-738.

6 Richard G, Lerche RC, Knospe V, Zermann H. Treatment of retinal arterial occlusion with local fibrinolysis using recombinant tissue plasminogen activator. Ophthalmology 1999; 106: 768-773.

7 Duker JS, Drown GC. Recovery following acute obstruction of the retinal and choroidal circulations: a case history. Retina 1988; 8: 257-260.

8 Hayreh SS, Zimmerman MB. Central retinal artery occlusion: visual outcome. Am J Ophthalmol 2005; 140: 376-391.

9 Arruga J, Sanders MD. Ophthalmologic findings in 70 patients with evidence of retinal embolism. Ophthalmology 1982; 89: 1336-1347. 